\title{
Lactic acid treatment of by-products and phosphorus level in the diet modulate bacterial microbiome and the predicted metagenome functions using the rumen simulation technique
}

\author{
Barbara U. Metzler-Zebeli, ${ }^{*}$ Andreas Haselmann,† Fenja Klevenhusen, ${ }^{*}$ Wilhelm Knaus, $\dagger$ \\ and Qendrim Zebeli*1 \\ *Institute of Animal Nutrition and Functional Plant Compounds, Department for Farm Animals and Veterinary Public Health, \\ University of Veterinary Medicine Vienna, Veterinärplatz 1, 1210 Vienna, Austria \\ †Department of Sustainable Agricultural Systems, Division of Livestock Sciences, BOKU-University of Natural Resources and Life Sciences, \\ 1180 Vienna, Austria
}

\begin{abstract}
This study used a rumen simulation technique to evaluate the effects of soaking of by-product-rich concentrate (BPC) in $5 \%$ lactic acid (LAC; vol/vol) on the rumen microbiota, predicted metagenome, fermentation characteristics, and nutrient degradation without or with supplemented P. The diet was supplemented with $1.6 \mathrm{~g}$ of $\mathrm{P}$ in the form of monocalcium phosphate per kilogram of dry matter in addition to $284 \mathrm{mg}$ of inorganic $\mathrm{P} / \mathrm{d}$ per fermentor via artificial saliva. Fermentor fluid was collected for analyses of short-chain fatty acids, fermentation gases, redox potential, and microbiota and feed residues for calculation of nutrient degradation. The microbiota composition was assessed using paired-end Illumina (Illumina Inc., San Diego, CA) MiSeq sequencing of the V3 to V5 region of the $16 \mathrm{~S}$ rRNA gene. Soaking in LAC reduced the contents of crude protein, neutral and acid detergent fibers, and organic matter fractions as well as ash and $\mathrm{P}$ content of the BPC. Both the LAC treatment of BPC and the inorganic $\mathrm{P}$ modified the relative bacterial abundances mainly within the predominant orders Bacteroidales and Clostridiales. Supervised DIABLO N-integration networking supported that operational taxonomic units related to BS11, Ruminococcaceae, Christensenellaceae, Eubacterium, and Selenomonas were the most discriminant for the LAC-treated BPC, whereas other operational taxonomic units related to BS11, RFN20, Ruminococcus, and Succiniclasticum were best correlated with the inorganic $\mathrm{P}$ supplementation. Integration networking also showed that carbohydrate and pyruvate metabolism, biosynthesis of unsaturated fatty acids, and degradation of several xenobiotics were stimulated by the LAC treatment of BPC. Those data sup-
\end{abstract}

Received March 26, 2018.

Accepted June 14, 2018.

${ }^{1}$ Corresponding author: Qendrim.Zebeli@vetmeduni.ac.at ported the enhanced fermentation activity as indicated by increased total short-chain fatty acid concentration, especially propionate and butyrate, and methane, but decreased ruminal crude protein degradation, with the LAC-treated compared with control-treated BPC. In contrast, despite an increased abundance of imputed functions, such as inositol phosphate metabolism, phosphatidylinositol signaling, and fructose and mannose metabolism, the reduced abundance of the imputed Kyoto Encyclopedia of Genes and Genomes pathway "transcription machinery" as well as the decrease in total short-chain fatty acids and nutrient degradation indicated reduced bacterial metabolic activity with the inorganic $\mathrm{P}$ supplementation. In conclusion, soaking of BPC in LAC may favor the proliferation of certain fibrolytic bacterial taxa and stimulate their metabolic activity, whereas the supplemented $\mathrm{P}$ to a diet already meeting ruminal $\mathrm{P}$ needs may impair ruminal nutrient utilization.

Key words: rumen microbiota, predicted metagenome function, lactic acid, phosphorus, by-product

\section{INTRODUCTION}

Aside from their cost efficiency, by-products from the food processing industry such as brans, middlings, oilseed products, and beet pulp have received special attention in ruminant nutrition for several reasons lately. Their utilization can limit the use of humanedible foods in diets for ruminants (Wilkinson and Lee, 2017). Moreover, cattle have the ability to transform large portions of human-inedible fibrous materials and plant-based proteins into animal proteins of superior quality (i.e., milk and meat; Ertl et al., 2016a). Byproducts differ from classical crops in such a way that they are commonly higher in dietary fiber and minerals and contain less starch or fat than original grains and legumes. Although research has confirmed their appropriateness in diets for ruminants (Hall and Chase, 2014; 
Ertl et al., 2016b), there is a dearth of information regarding the effects of by-product-rich diets on the rumen microbial ecosystem, especially when feeding them as the sole concentrate source to cattle. Therefore, it is important to exploit their effect on the rumen microbial homeostasis to avoid rumen dysbiosis and to optimize their nutritional value for production efficiency reasons. Particularly, the high $\mathrm{P}$ content in by-products may be attractive for cattle nutrition. However, the $\mathrm{P}$ in these by-products is mostly bound as phytate-P, which is less ruminally available (Humer and Zebeli, 2015). Regardless of the microbial phytase activity in the rumen (Clark et al., 1986; Morse et al., 1992; Feng et al., $2013)$, the ruminal degradation of phytate-P is often incomplete, especially under the intense feeding regimens in modern dairy production (Khol-Parisini et al., 2015), limiting the phytate-P availability for microbes and the host. We have previously shown that soaking of barley grain with 5\% lactic acid (LAC) was able to enhance the phytate-P availability and to replace inorganic $\mathrm{P}$ to meet microbial $\mathrm{P}$ needs for bacterial growth and fermentation (Metzler-Zebeli et al., 2014; Harder et al., 2015). Therefore, treating by-products with LAC may be interesting from an environmental point of view as less inorganic $\mathrm{P}$ needs to be supplemented to diets for cattle. The LAC-treated barley also had an increased hemicellulose content and decreased ruminal degradability of starch (Harder et al., 2015; Khol-Parisini et al., 2015), which, together with the reduced phytate-P content, altered the ruminal abundance of important amylolytic and fibrolytic taxa, such as fungi, Lactobacillus, Ruminococcus albus and Ruminococcus flavefaciens, and Megasphaera elsdenii (Metzler-Zebeli et al., 2015; Deckardt et al., 2016). Due to the differences in nutrient composition, the effects of soaking by-products in LAC on the ruminal microbiota can be expected to differ from those of cereal grains. Because by-products are richer in dietary fiber than cereal grains, we hypothesized that the LAC treatment of by-products would especially modulate the fibrolytic bacterial community, which may benefit from an increased $\mathrm{P}$ availability in the rumen due to the greater bacterial $\mathrm{P}$ requirement to break down fiber (Durand and Komisarczuk, 1988). Despite sufficient dietary $\mathrm{P}$ from organic sources, it is general practice to supplement high-producing cattle diets with inorganic $\mathrm{P}$ to compensate the incomplete ruminal phytate-P hydrolysis and ensure sufficient amounts of ruminally available P (Humer and Zebeli, 2015). We therefore further hypothesized that the LAC treatment of by-products has the same effect on the rumen microbiota and their metabolic activity as adding inorganic P sources.

The objective was to evaluate the effect of soaking a by-product-rich concentrate (BPC) with LAC and supplementing with or without inorganic $\mathrm{P}$ on the rumen microbiome, the predicted bacterial metagenome, fermentation characteristics, and nutrient degradation using the rumen simulation technique (Rusitec).

\section{MATERIALS AND METHODS}

\section{Processing of BPC and Experimental Treatments}

The BPC consisted solely of industrial by-products, including corn gluten feed (31.8\%), beet pulp (31.5\%), sunflower cake $(18.6 \%)$, and wheat bran $(18.2 \%)$. Chemical composition of the BPC was $93.8 \%$ DM, $6.16 \%$ ash, $19.4 \%$ CP, $4.6 \%$ ether extract, $48.8 \%$ NDF, $20.9 \% \mathrm{ADF}$, and $0.69 \%$ total P (DM basis). Four identical diets were prepared, with the only difference in the treatment of the BPC and inorganic $\mathrm{P}$ supplementation (Supplemental Table S1; https://doi.org/10.3168/jds .2018-14821). The processing procedure was similar to that of Harder et al. (2015) and consisted of soaking the BPC 1:5 (wt/vol) in either distilled water as control $(\mathbf{C O N})$ or in a $5 \%$ LAC solution (vol/vol; DL-lactate, 80\% wt/wt, Brenntag CEE GmbH, Vienna, Austria) for $24 \mathrm{~h}$. The $\mathrm{pH}$ of the $5 \%$ LAC solution was 1.8 before the soaking. After the soaking procedure, the $\mathrm{CON}$ and LAC-treated BPC were spread onto Petri dishes $(150 \times$ $25 \mathrm{~mm}$ ) and dried at $40^{\circ} \mathrm{C}$ for $96 \mathrm{~h}$.

The different $\mathrm{P}$ levels were produced via different $\mathrm{P}$ levels in the dietary mineral premix and the buffer composition. Four total mixed diets were formulated (Supplemental Table S1; https://doi.org/10.3168/jds.2018 -14821) that consisted of hay and grass silage as forage sources $(50 \%$ of $\mathrm{DM})$, and the CON- or LAC-treated $\mathrm{BPC}(48 \%$ of DM), as well as the $2 \%$ of DM as mineral premix (Biomin M16, Biomin GmbH, Herzogenburg, Austria). The latter was either without or with an additional inorganic $\mathrm{P}$ source in form of monocalcium phosphate (Supplemental Table S1). Total P content differed $1.6 \mathrm{~g} / \mathrm{kg}$ of DM between the nonsupplemented and P-supplemented diets. In addition to the different dietary P levels, 2 artificial saliva buffers with different $\mathrm{P}$ levels were prepared. The buffer without inorganic $\mathrm{P}$ was prepared according to Harder et al. (2015), keeping a similar estimated osmolarity as the McDougall buffer (343 vs. 346 mOsm; McDougall, 1948). The buffer with inorganic $\mathrm{P}$ was prepared according to McDougall (1948), providing additionally $0.284 \mathrm{~g}$ of $\mathrm{P}$ per day for each vessel.

\section{Experimental Procedure and Sampling}

The experiment was conducted in 2 identical experimental runs. Each run lasted $10 \mathrm{~d}$ with an adaptation 
period of $5 \mathrm{~d}$, allowing for equilibration of the system, followed by $5 \mathrm{~d}$ for samplings. The experiment was designed as a randomized $2 \times 2$ factorial design with the treatment of $\mathrm{BPC}$ (i.e., $\mathrm{CON}$ or $\mathrm{LAC}$ ) and inorganic $\mathrm{P}$ supplementation (supplemented or not) as the main factors. This resulted in 4 treatments tested in 3 fermentors in each run, and 6 independent measurements per treatment.

To prepare the inocula in each run, ventral ruminal fluid and solid digesta were collected from 3 nonlactating rumen-fistulated Holstein cows, housed at the Teaching and Research Farm Kremesberg of the University of Veterinary Medicine, Vienna, Austria. Cows were fed with $33.3 \%$ hay and $66.6 \%$ silage (DM basis) ad libitum and were kept according to the Austrian guidelines for animal welfare (Federal Ministry of Health, 2004). The preparation, inoculation of the system, and daily feeding procedures were essentially done as described before (Harder et al., 2015), with an infusion rate of artificial saliva of $350 \mathrm{~mL} / \mathrm{d}( \pm 50)$ using a 12-channel peristaltic pump (IPC-N 12/ISM 937, IDEX Health \& Sciences $\mathrm{GmbH})$. The mixed feed was weighed into a nylon bag $(70 \times 140 \mathrm{~mm}, 150 \mu \mathrm{m}$ mesh size $) ; 2$ feed-containing nylon bags were incubated for $48 \mathrm{~h}$ per fermentor.

Fluid samples from each fermentor were collected daily directly before the feeding procedure to measure pH (In Lab Expert Pro-ISM pH, Mettler Toledo), redox potential (Pt 4805-DPA-SC-S8/120, Mettler Toledo), and ammonia concentration, using a syringe inserted through a 3 -way valve. For this, a digital $\mathrm{pH}$ meter was used, with calibration buffers of $\mathrm{pH} 4.0$ and 7.0. Besides collecting fermentor fluid samples for $\mathrm{pH}$ and redox potential measurements from d 6 to 10, fluid samples of each fermentor $(1.8 \mathrm{~mL})$ were taken, frozen at $-20^{\circ} \mathrm{C}$ for analysis of short-chain fatty acids (SCFA) and ammonia, and snap-frozen and stored at $-80^{\circ} \mathrm{C}$ for the microbiota composition. Gas-tight aluminum bags (Tecobag $5 \mathrm{~L}$, Tesseraux Spezialverpackungen $\mathrm{GmbH}$, Bürstadt, Germany) were used for collection of fermentation gases over $24 \mathrm{~h}$ on each sampling day to determine $\mathrm{CH}_{4}$ and $\mathrm{CO}_{2}$ concentrations (ATEX Biogas Monitor Check BM 2000, Ansyco, Karlsruhe, Germany). The total gas amount was quantified using the water displacement technique (Deckardt et al., 2016). Moreover, samples for ammonia and SCFA containing the residues were stored at $-20^{\circ} \mathrm{C}$ and at $-80^{\circ} \mathrm{C}$ for the microbiota composition until analyses.

Additionally, after recovery from the fermentor, the nylon bags were gently rinsed with tap water. The bags were washed for 2 min until the liquid ran clear (Deckardt et al., 2016). After washing, bags were stored at $-20^{\circ} \mathrm{C}$ before air oven drying $\left(60^{\circ} \mathrm{C}\right.$, overnight). Thereafter, samples were acclimatized to air and weighed. Residues collected on d 6 to 10 were pooled for each fermentor, and ground to pass a 1-mm screen (Ultra Centrifugal Mill ZM 200, Retsch GmbH, Haan, Germany).

\section{Chemical Analysis}

The ammonia concentration in fermentor fluid of each sampling day (d 6 to 10) was determined spectrophotometrically (UV-1800, Shimadzu Corporation, Kyoto, Japan) by measuring the light absorbance at $655 \mathrm{~nm}$ according to the indophenol method (Weatherburn, 1967). The concentrations of SCFA (acetate, propionate, butyrate, isobutyrate, valerate, isovalerate, and caproate) in the fermentor fluid were measured after pooling the single samples of each fermentor from d 6 to 10. Preparation of the samples and analysis of the SCFA concentration was performed as described previously and molar proportions of individual SCFA were calculated (Qumar et al., 2016).

Feed components and nylon bag residues were analyzed for DM (method 3.1; Naumann and Basler, 2012), ash (method 8.1; Naumann and Basler, 2012), N using the Kjeldahl method (method 4.1.1; Naumann and Basler, 2012), ether extract (method 5.1.1B; Naumann and Basler, 2012), NDF (with heat-stable $\alpha$-amylase; Van Soest et al., 1991), and ADF using an Ankom 2000 Fiber Analyzer (Ankom Technology Corp., Macedon, NY). The NDF is expressed as alpha amylase-stable neutral detergent fiber exclusive of residual ash (aND$\mathbf{F}_{\text {OM }}$ ), whereas the ADF is expressed as acid detergent fiber exclusive of residual ash $\left(\mathbf{A D F}_{\mathrm{OM}}\right)$. The total $\mathrm{P}$ concentration was determined spectrophotometrically using the vanadate-molybdate method (method 10.6.1; Naumann and Basler, 2012), measuring the light absorbance at $436 \mathrm{~nm}$ after wet-ashing with $\mathrm{HNO}_{3}$ and $\mathrm{H}_{2} \mathrm{O}_{2}$ in a microwave (MLS-ETHOS plus Terminal 320, Leutkirch, Germany; method 10.6.1; Naumann and Basler, 2012). Nutrient degradation was calculated as the difference between the nutrients of the fed experimental diet and those in the feed residues after $48 \mathrm{~h}$ of incubation.

\section{DNA Extraction and Absolute Quantification}

For the genomic DNA isolation, fermentor samples from the collection d 6 to 10 were thawed on ice and pooled. An aliquot $(300 \mu \mathrm{L})$ of the pooled fermentor samples were processed for genomic DNA extraction using a modified protocol of the PowerSoil DNA isolation kit (MoBio Laboratories Inc., Carlsbad, CA) as described in Metzler-Zebeli et al. (2015). The DNA concentration in each DNA extract was quantified using the Qubit 2.0 Fluorometer (Life Technologies, Carlsbad, CA) and the Qubit dsDNA HS Assay Kit (Life 
Technologies). The identical DNA sample was used for 16S rRNA gene amplicon sequencing and quantitative PCR analysis. For the quantitative PCR, DNA sample volumes were adjusted to achieve similar DNA concentrations across samples to avoid an effect of the DNA concentration on the amplification results.

Primer sets used for quantitative PCR of the bacterial and archaeal $16 \mathrm{~S}$ rRNA genes, the protozoal $18 \mathrm{~S}$ rRNA gene and anaerobic fungi (5.8 and 18S rRNA gene) have been described previously (Metzler-Zebeli et al., 2015). Amplifications were performed on a Stratagene Mx3000P QPCR System (Agilent Technologies, Santa Clara, CA) using the Fast-Plus EvaGreen Master Mix with Low ROX (Biotium, Hayward, CA). Each standard and sample reaction contained $10 \mu \mathrm{L}$ of master mix, forward and reverse primers $(62.5 \mathrm{pmol})$, and $4 \mathrm{ng}$ of DNA template. An initial denaturation step at $95^{\circ} \mathrm{C}$ for 5 min was followed by 40 cycles of $95^{\circ} \mathrm{C}$ for 15 $\mathrm{s}$, primer annealing at $60^{\circ} \mathrm{C}$ for $30 \mathrm{~s}$, and elongation at $72^{\circ} \mathrm{C}$ for $30 \mathrm{~s}$. Fluorescence was measured at the last step of each cycle. The dissociation of PCR products was monitored by slow heating with an increment of $0.5^{\circ} \mathrm{C} / \mathrm{s}$ from 55 to $95^{\circ} \mathrm{C}$ to determine the specificity of the amplification. Amplification efficiency was calculated according to $\mathrm{E}=-1+10^{-1 / \text { slope }}$. The standards were created from the purified and quantified PCR products generated by standard PCR using DNA from fermentor fluid of the present study and the respective bacterial primer set. Ten-fold standard serial dilutions $\left(10^{7}\right.$ to $10^{3}$ molecules $\left./ \mu \mathrm{L}\right)$ were run on each 96 -well plate, with amplification efficiencies ranging from 1.89 to 1.94 and $\mathrm{R}^{2}=0.99$ to 1.00 .

\section{S rRNA Sequencing, Taxonomic Classification, and Diversity Analysis}

An aliquot of each of the extracted DNA samples was sent for PCR amplification of the 16S rRNA gene, library preparation, and DNA sequencing to a commercial provider (Microsynth AG, Balgach, Switzerland). Primers 357F-HMP (5'-CCTACGGGAGGCAGCAG-3') and 926R-HMP (5'-CCGTCAATTCMTTTRAGT-3') targeting the V3 to V5 region of the 16S rRNA gene were used for amplification, generating an approximate amplicon size of $570 \mathrm{bp}$ (Peterson et al., 2009). The $16 \mathrm{~S}$ rRNA gene PCRs were performed using the KAPA HiFi HotStart PCR Kit (Roche, Baden, Switzerland). Libraries were constructed by ligating sequencing adapters and indices onto purified PCR products using the Nextera XT sample preparation kit (Illumina Inc., San Diego, CA). Equimolar quantities of each library were pooled and sequenced on an Illumina MiSeq sequencing v2 platform using a 300- bp read length paired-end protocol. After sequencing, the overlapping paired-end reads were demultiplexed, trimmed of Illumina adaptor residuals using cutadapt (code.google.com/p/cutadapt/), and stitched using USEARCH (drive5/com) by Microsynth. In total, $1,056,857$ stitched reads for the 24 fermentor samples with a mean Phred score of 35 were obtained from the commercial provider.

Stitched reads were processed using Quantitative Insights Into Microbial Ecology (QIIME, v1.9.4; Caporaso et al., 2010). Following removal of low quality sequences $(\mathrm{q}<15)$ and assembly, chimeric sequences were filtered out with the UCHIME method using USEARCH8.1 and gold.fa database (Edgar, 2010; Edgar et al., 2011). Open-reference operational taxonomic unit (OTU) picking was done at $97 \%$ similarity level using UCLUST (Edgar, 2010) with a minimum cluster size of 10 . The most abundant sequence was selected from each OTU cluster and taxonomically assigned using the Ribosomal Database Project (RDP) classifier tool (Cole et al., 2009) with the most recent version of the Greengenes database (13.8; http://greengenes .secondgenome.com/). Measures of microbial richness and diversity were calculated using the nonparametric species estimator Chao1 as well as the Shannon and Simpson's indices of diversity in QIIME and were based on a rarefaction depth of 20,000 reads per sample. To determine the degree of similarity between samples, weighted and unweighted UniFrac distance matrices were calculated. Identified key OTU were additionally classified against the National Center for Biotechnology Information (NCBI) nucleotide database using Microbial Nucleotide BLAST (https://blast.ncbi.nlm .nih.gov/). The raw sequence reads were uploaded to the NCBI Bioproject databank under the Bioproject number: PRJNA439186.

\section{Microbial Metagenome Prediction}

Microbial metagenome prediction for each sample based on 16S rRNA gene sequencing data was determined using Phylogenetic Investigation of Communities by Reconstruction of Unobserved States (PICRUSt; Langille et al., 2013). For this, closed-reference OTU picking was performed at $97 \%$ similarity level against the Greengenes database (downloaded from http:// greengenes.secondgenome.com/downloads/database/ 13_5), and processed in the online Galaxy PICRUSt interface (http://galaxyproject.org/) with a workflow described by the developers (http://picrust.github.com/ picrust/tutorials/quickstart.html\#quickstartguide). The sequences were categorized by function based on Kyoto Encyclopedia of Genes and Genomes (KEGG) 
pathways in PICRUSt. Non-bacteria-related KEGG orthology functions and functions $<0.01 \%$ relative abundance were dismissed.

\section{Statistical Analyses}

Daily measured data were averaged over the sampling period. To test for differences in relative abundance of bacterial taxa, only taxa appearing in at least $75 \%$ of the samples were considered. The raw read counts from the tables of OTU abundances and predicted KEGG pathways were collapsed at taxonomic rank and KEGG pathway and compositionally and functionally normalized such that each sample sums to 1 , respectively. The relative abundances (referred to as relative abundance from this point forward in the text) of the bacterial populations and KEGG pathways at the respective taxonomic and functional rank were analyzed.

Data of fermentation characteristics, nutrient degradation, microbial gene copy numbers, and relative abundances of bacterial microbiota and predicted KEGG pathways were first analyzed for normality using Shapiro-Wilk test with the UNIVARIATE procedure in SAS (version 9.4, SAS Institute Inc., Cary, NC). After establishing normality, fermentation and nutrient degradation data and microbial gene copy numbers were subjected to ANOVA using the MIXED procedure in SAS. Regarding the bacterial microbiota and predicted KEGG pathways, significance between treatment groups was assessed with ANOVA for data at phyla and family level, the most abundant OTU (only the first 20 significantly different OTU are presented), and the most discriminant OTU and KEGG pathways identified using DIABLO N-integration analysis. Fixed effects included in the model were treatment with LAC, inorganic P supplementation, and their 2-way interaction. The fermentor and the run were considered as random effects. If interactions were present between the fixed effects, least squares means were compared with the Tukey-Kramer test. For testing the hypothesis, the level of significance was set at $P<0.05$. The false discovery rate was applied on raw $P$-values of the most abundant and discriminant bacterial OTU and predicted KEGG pathways (Benjamini and Hochberg, 1995) using the MULTTEST procedure (SAS). False discovery rate-corrected $P$-values below 0.05 were considered significant.

To identify the most discriminant OTU and predicted metabolic functions, supervised DIABLO N-integration networking was performed using the package mixOmics (version 6.3; Rohart et al., 2017) in R studio. Horizontal sparse partial least squares-discriminant analysis (sPLS-DA) was used to integrate the data sets of relative abundances of OTU with those of predicted KEGG pathways to classify and select key features from each data set. In general, DIABLO N-integration allows for accounting of the heterogeneity between data sets measured on the same samples (e.g., "omics" platforms), thereby considering the relationships between different functional levels (Rohart et al., 2017). Tuning of sPLS-DA parameters was performed to determine the main OTU and predicted KEGG pathways that enable discrimination of treatments groups with the lowest possible error rate, selecting 20 OTU and 10 KEGG pathways each for components 1 and 2, respectively. The sPLS-DA results were visualized as scatter plots for individuals (experimental units) per data set, clustered image maps (Euclidean distance, complete linkage), and circus plots showing the strongest positive and negative Pearson correlations $(|\mathrm{r}|>0.8)$ between most discriminant OTU and KEGG pathways for each subset of data and identified features. After identification of the main OTU that enable discrimination of soaking treatment of $\mathrm{BPC}$ and supplementation of inorganic $\mathrm{P}$, these discriminant OTU were additionally correlated with the data of fermentation and nutrient degradation using the package "corrplot" and library "RColorBrewer" in R.

\section{RESULTS}

\section{Lactic Acid Treatment of By-Products and Fermentation Characteristics}

Treating the BPC with LAC reduced the contents of ash, $\mathrm{CP}$, ether extract, $\mathrm{aNDF}_{\mathrm{OM}}$, and $\mathrm{ADF}_{\mathrm{OM}}$, and $\mathrm{P}$ by $19.5,13.7,11.8,13.218 .3$, and $13.0 \%$, whereas the content of NFC increased by $53.8 \%$ in the LAC-treated BPC compared with the CON-treated BPC (Table 1). Although statistically different, the effects of the LAC treatment of BPC and inorganic $\mathrm{P}$ supplementation on rumen $\mathrm{pH}$ were small $(P<0.05$; Table 1$)$. The LAC treatment of the BPC decreased $(P<0.05)$ the ammonia concentration as well as the redox potential in the fermentor fluid by 18.4 and $5.1 \%$ compared with the CON treatment, respectively. The daily production of methane and $\mathrm{CO}_{2}$, however, increased $(P<0.05)$ by 8.2 and $16.5 \%$ with the LAC treatment of BPC compared with the CON treatment, respectively. Moreover, the inorganic $\mathrm{P}$ supplementation reduced $(P<0.05)$ the ammonia concentration, the redox potential, as well as methane and $\mathrm{CO}_{2}$ generation by $4.4,6.2,6.8$, and $13.7 \%$ compared with the nonsupplemented fermentors, respectively. With regard to the degradation of nutrients in the fermentors, the LAC treatment of BPC only decreased $(P<0.05)$ the degradation of $\mathrm{CP}$ by $4.0 \%$ compared with the CON treatment. The additional inorganic $\mathrm{P}$ decreased $(P<0.05)$ the degradation of 
$\mathrm{OM}$, ash, $\mathrm{CP}$, ether extract, $\mathrm{aNDF}_{\mathrm{OM}}, \mathrm{ADF}_{\mathrm{OM}}$, and total $\mathrm{P}$ by $4.7,3.8,13.4,5.5,9.8$, and $5.3 \%$ compared with the nonsupplemented fermentors, respectively. The degradation of NFC, in turn, was enhanced $(P$ $<0.05$ ) by $2 \%$ with the extra inorganic $\mathrm{P}$ and almost reached $100 \%$ compared with the fermentors without inorganic P supplementation. The LAC treatment of BPC enhanced the total SCFA concentration by $10.3 \%$ compared with the CON treatment (Table 1), whereas the inorganic $\mathrm{P}$ supplementation reduced total SCFA in the fermentor fluid by $5.7 \%(P<0.001)$. The molar proportions of individual SCFA changed to less acetate, valerate, caproate, and branched-chain fatty acids and to more propionate and butyrate with the $\mathrm{LAC}$ treated BPC compared with the CON treatment $(P<0.001)$. This decreased $(P<0.001)$ the acetate-to-propionate ratio by $27 \%$ with the LAC-treated compared with the CON-treated BPC diet. The additional inorganic $\mathrm{P}$, in turn, increased the proportion of valerate and isovalerate to the expense of acetate $(P<0.05)$.

\section{Sequencing Details and Characterization of Bacterial Microbiota}

After quality control and chimera check, a total of 876,724 sequencing reads with an average number of merged sequences reads per sample of 28,148 reads and a mean read length of $540 \mathrm{bp}$ were obtained for the 24 fermentor fluid and original rumen inoculum samples. Using a dissimilarity threshold of $3 \%$ sequence divergence to classify OTU and a relative abundance threshold of $0.01 \%, 1,016$ distinct OTU in the fermentor fluid samples could be identified. Representative sequences from the OTU were assigned to 17 bacterial phyla and 60 families (Supplemental Table S2; https:/ /doi.org/10.3168/jds.2018-14821). Phylum-level analysis showed that 6 phyla dominated, with Firmicutes and Bacteroidetes representing about 53 and $28 \%$ of all sequences, respectively, followed by Spirochaetes $(3.6 \%)$, Tenericutes $(3.0 \%)$, Proteobacteria $(2.6 \%)$, and Verrucomicrobia (1.5\%; Figure 1). Phylogenetic clas-

Table 1. Differences in fermentation traits, short-chain fatty acids (SCFA), and nutrient degradation of diets containing either the control $(\mathrm{CON})$ - or $5 \%$ lactic acid (LAC)-treated by-product-rich concentrate without $(-\mathrm{P})$ or with $(+\mathrm{P})$ inorganic $\mathrm{P}$ supplementation $^{1}$

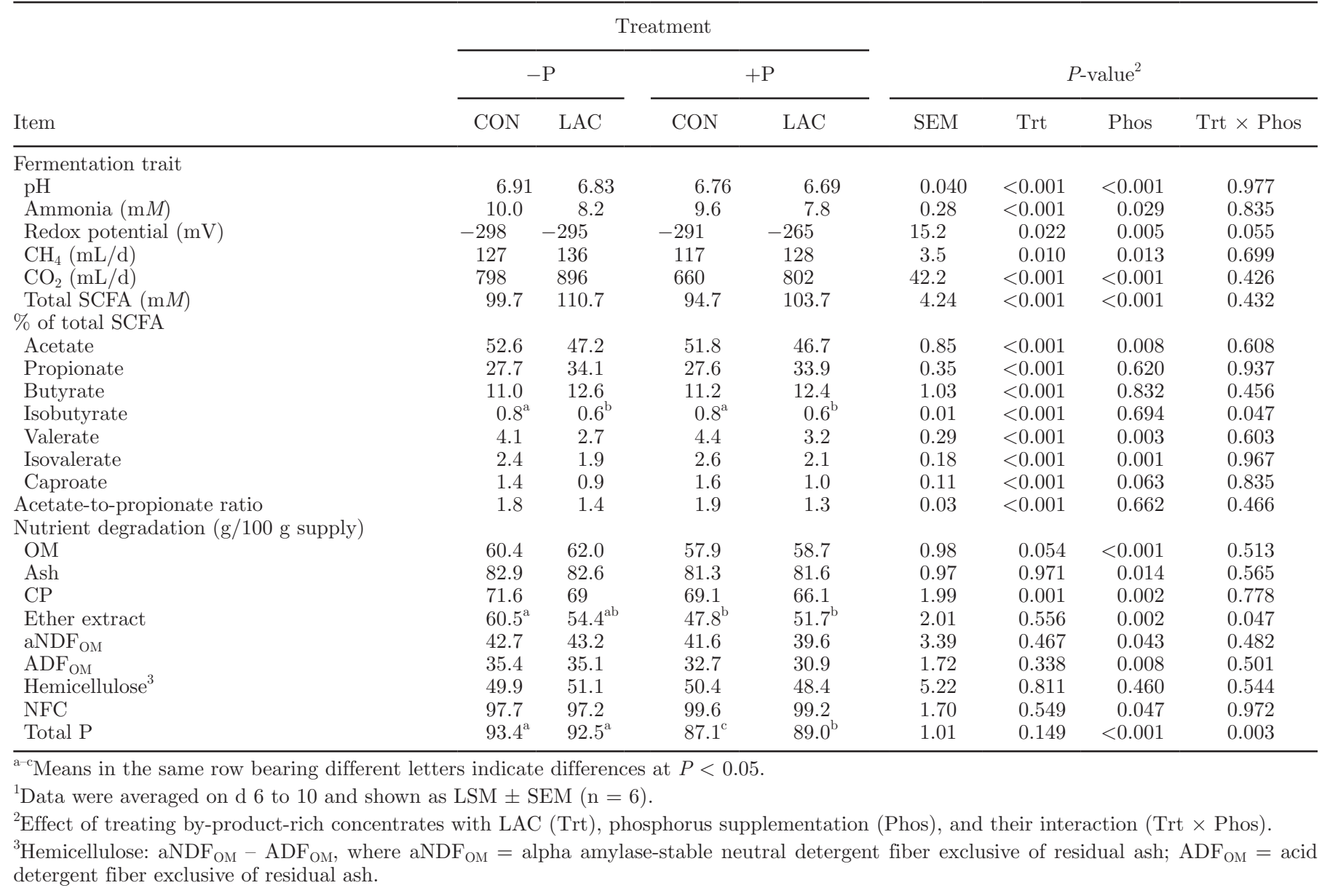




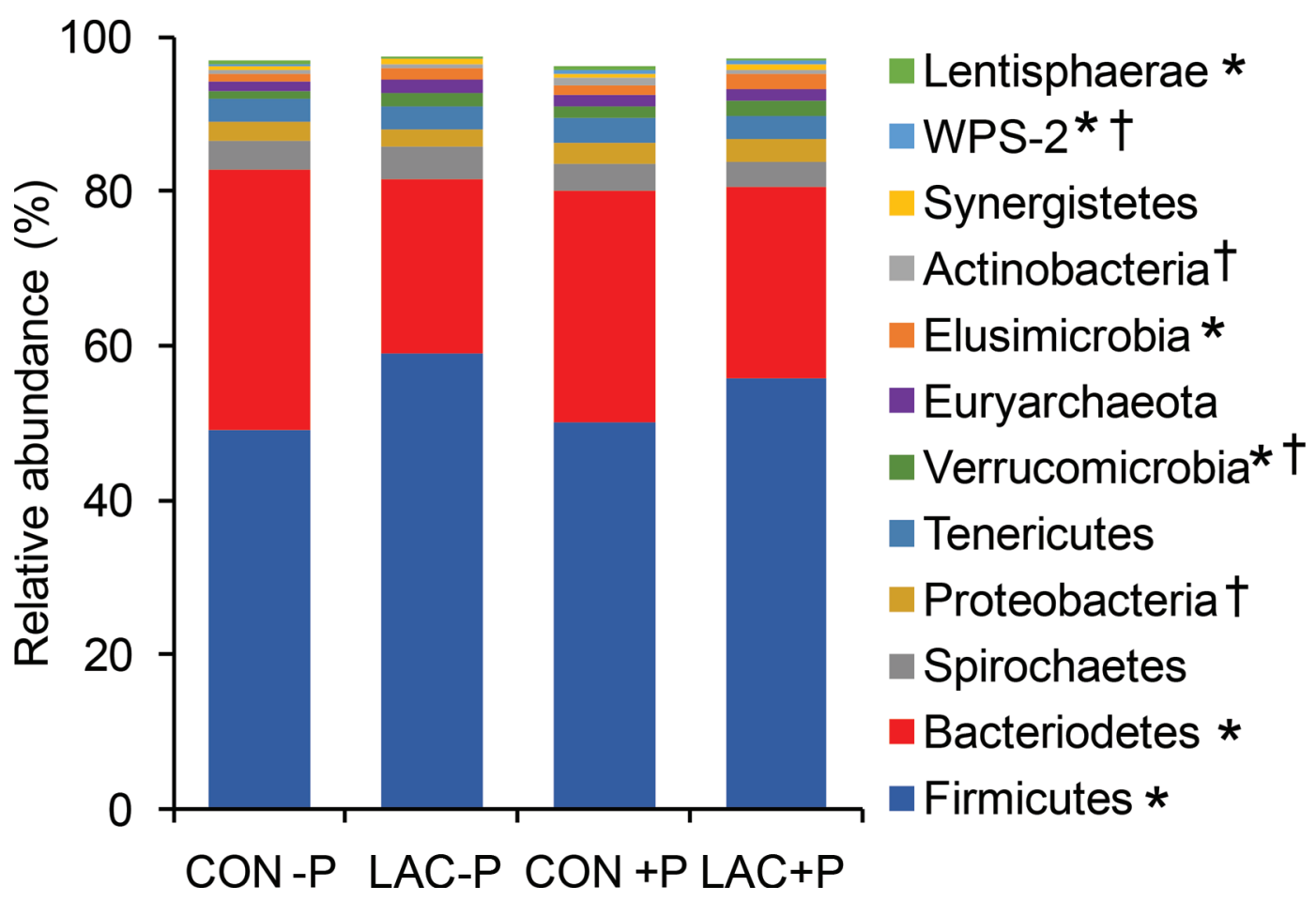

Figure 1. Relative abundance (\% of all reads) of bacterial phyla in fermentor fluid incubated with diets containing either the control (CON)or lactic acid (LAC)-treated by-product-rich concentrate without $(-\mathrm{P})$ or with $(+\mathrm{P})$ inorganic $\mathrm{P}$ supplementation. ${ }^{*} P<0.05$, effect of lactic acid; $† P<0.05$, effect of $\mathrm{P}$ level. $\mathrm{n}=6$ per treatment. Color version available online.

sification identified 41 families with a mean abundance greater than $0.05 \%$ of all reads (Table 2). The Bacteroides mainly encompassed the family $B S 11$ and an unclassified Bacteroidales family with $24.2 \%$ and $3.6 \%$ of all reads, respectively, whereas the Firmicutes community was dominated by the families Ruminococcaceae (21.5\% of all reads), an unclassified Clostridiales family 1 (21.5\% of all reads), and Veillonellaceae ( $5.6 \%$ of all reads).

\section{Treatment Effects on Bacterial Community Structure and Composition}

Both the LAC treatment of the BPC and the inorganic $\mathrm{P}$ supplementation affected the bacterial species richness and evenness (Table 3). As such, the LAC treatment of the BPC increased the number of observed OTU as well as the Simpson and Shannon indices $(P$ $<0.01$ ), whereas the added inorganic $\mathrm{P}$ decreased the Chao1 but increased the Simpson index compared with the treatment without inorganic $\mathrm{P}(P<0.05)$. The LAC treatment of BPC and the supplemented inorganic $\mathrm{P}$ did not affect the absolute abundance of total bacteria, archaea, and protozoa. However, both treatments increased the gene copy number of fungi by $0.2 \log$ units, though the LAC effect was mainly obvious in fermentors without the additional inorganic $\mathrm{P}(P<0.05$; Table 3$)$. With respect to the relative bacterial abundances, diet-related shifts were detectable for almost all predominant phyla (Figure 1). Correspondingly, LAC treatment of BPC increased $(P<$ 0.05 ) the relative abundance of the phyla Firmicutes, Verrucomicrobia, and Elusimicrobia by 16, 58, and 64\%, respectively, compared with the CON-treated BPC. In contrast, the LAC treatment decreased the relative abundance of Bacteroidetes, WPS-2, and Lentisphaerae by 25,29 , and $12 \%$ compared with the CON treatment, respectively, with the effects being stronger without the inorganic $\mathrm{P}$ for Bacteroidetes as indicated by the LAC $\times$ inorganic $\mathrm{P}$ interaction $(P<0.05)$. The supplemented inorganic $\mathrm{P}$, in turn, decreased the abundance of Proteobacteria, whereas it promoted Verrucomicrobia, Actinobacteria, and WPS-2 $(P<0.05)$.

In total, the LAC treatment of BPC and the inorganic $\mathrm{P}$ supplementation modified the abundance of 19 families each (Table 2). Within the Firmicutes, the LAC treatment of BPC increased the relative abundance of the families Ruminococcaceae, Clostridiales 1, Christensenellaceae by 14, 17, and 108\%, respectively, and decreased $(P<0.05)$ that of Clostridiaceae by $29 \%$ compared with the CON-treated BPC. It further decreased the most predominant family BS11 and an- 
Table 2. Ruminal differences in relative abundances (\% of all reads) of bacterial families in fermentor liquid incubated with diets containing either the control (CON)- or $5 \%$ lactic acid (LAC)-treated by-product-rich concentrate without $(-\mathrm{P})$ or with $(+\mathrm{P})$ inorganic $\mathrm{P}$ supplementation

\begin{tabular}{|c|c|c|c|c|c|c|c|c|}
\hline Family & \multicolumn{4}{|c|}{ Treatment } & SEM & \multicolumn{3}{|c|}{$P$-value ${ }^{1}$} \\
\hline$B S 11$ & 29.47 & 19.50 & 26.10 & 21.75 & 4.015 & $<0.001$ & 0.579 & 0.084 \\
\hline Ruminococcaceae & $19.08^{\mathrm{b}}$ & $23.06^{\mathrm{a}}$ & $21.11^{\mathrm{ab}}$ & $22.62^{\mathrm{a}}$ & 1.170 & $<0.001$ & 0.149 & 0.037 \\
\hline Clostridiales family 1 & $18.55^{\mathrm{b}}$ & $22.72^{\mathrm{a}}$ & $16.86^{\mathrm{b}}$ & $18.64^{\mathrm{b}}$ & 0.495 & $<0.001$ & $<0.001$ & 0.021 \\
\hline Christensenellaceae & 1.50 & 3.41 & 1.68 & 3.19 & 0.507 & $<0.001$ & 0.865 & 0.498 \\
\hline ML615J-28 family & 1.77 & 1.78 & 2.25 & 2.00 & 0.130 & 0.227 & 0.005 & 0.427 \\
\hline RF39 family & 0.97 & 1.08 & 0.81 & 0.96 & 0.046 & 0.014 & 0.011 & 0.712 \\
\hline$R_{4}-41 B$ & 0.61 & 1.45 & 0.85 & 1.47 & 0.354 & $<0.001$ & 0.100 & 0.438 \\
\hline Endomicrobia & 0.31 & 1.03 & 0.52 & 1.39 & 0.482 & $<0.001$ & 0.064 & 0.773 \\
\hline RF32 family & 0.37 & 0.27 & 0.44 & 0.40 & 0.166 & 0.125 & 0.040 & 0.496 \\
\hline Elusimicrobiaceae & 0.58 & 0.50 & 0.67 & 0.51 & 0.055 & 0.032 & 0.309 & 0.402 \\
\hline$W P S-2$ family & 0.44 & 0.19 & 0.55 & 0.51 & 0.079 & 0.037 & 0.004 & 0.241 \\
\hline Rickettsiales family & 0.10 & 0.14 & 0.15 & 0.19 & 0.021 & 0.087 & 0.035 & 0.996 \\
\hline Mollicutes family & 0.15 & 0.07 & 0.17 & 0.10 & 0.019 & $<0.001$ & 0.020 & 0.847 \\
\hline$R_{4}-45 B$ & 0.10 & 0.09 & 0.12 & 0.09 & 0.027 & 0.015 & 0.154 & 0.393 \\
\hline Synergistaceae & 0.11 & 0.09 & 0.11 & 0.07 & 0.037 & 0.025 & 0.435 & 0.157 \\
\hline GMD14H09 family & 0.038 & 0.111 & 0.046 & 0.105 & 0.020 & 0.001 & 0.934 & 0.710 \\
\hline Enterobacteriaceae & $0.078^{\mathrm{a}}$ & $0.058^{\mathrm{ab}}$ & $0.027^{\mathrm{c}}$ & $0.065^{\mathrm{ab}}$ & 0.009 & 0.347 & 0.041 & 0.012 \\
\hline$R B 046$ family & 0.024 & 0.033 & 0.043 & 0.037 & 0.015 & 0.798 & 0.039 & 0.156 \\
\hline Burkholderiales & 0.008 & 0.021 & 0.006 & 0.009 & 0.005 & 0.018 & 0.028 & 0.094 \\
\hline Anaerolinaceae & 0.038 & 0.047 & 0.031 & 0.031 & 0.005 & 0.366 & 0.033 & 0.377 \\
\hline$F 16$ & 0.033 & 0.038 & 0.021 & 0.017 & 0.010 & 0.850 & 0.012 & 0.470 \\
\hline Pirellulaceae & 0.035 & 0.037 & 0.026 & 0.019 & 0.008 & 0.687 & 0.042 & 0.421 \\
\hline PB19 family & 0.020 & 0.022 & 0.008 & 0.012 & 0.004 & 0.345 & 0.004 & 0.731 \\
\hline
\end{tabular}

${ }^{\mathrm{a}-c}$ Means in the same row bearing different letters indicate differences at $P<0.05$.

${ }^{1}$ Effect of treating by-product-rich concentrates with LAC (Trt), phosphorus supplementation (Phos), and their interaction (Trt $\times$ Phos).

Table 3. Differences in $\alpha$-diversity indices and microbial gene copy numbers $\left(\log _{10}\right.$ gene copies $\left./ \mathrm{mL}\right)$ in fermentor liquid incubated with diets containing either the control $(\mathrm{CON})$ - or $5 \%$ lactic acid (LAC)-treated by-product-rich concentrate without $(-\mathrm{P})$ or with $(+\mathrm{P})$ inorganic $\mathrm{P}$ supplementation

\begin{tabular}{|c|c|c|c|c|c|c|c|c|}
\hline Item & \multicolumn{4}{|c|}{ Treatment } & SEM & \multicolumn{3}{|c|}{$P$-value ${ }^{1}$} \\
\hline$\overline{\text { Observed OTU }^{2}}$ & 1,837 & 1,936 & 1,835 & 1,876 & 17.64 & 0.003 & 0.106 & 0.124 \\
\hline Chao1 & 2,619 & 2,715 & 2,612 & 2,641 & 31.78 & 0.238 & 0.080 & 0.324 \\
\hline Simpson & 0.97 & 0.99 & 0.98 & 0.99 & 0.009 & 0.001 & 0.040 & 0.261 \\
\hline \multicolumn{9}{|c|}{$\log _{10}$ gene copies $/ \mathrm{mL}$} \\
\hline Total bacteria & 9.1 & 9.1 & 9.2 & 9.2 & 0.07 & 0.583 & 0.062 & 0.472 \\
\hline Archaea & 6.8 & 6.7 & 6.8 & 6.8 & 0.04 & 0.453 & 0.106 & 0.941 \\
\hline Protozoa & 6.8 & 6.9 & 6.9 & 6.8 & 0.17 & 0.922 & 0.459 & 0.215 \\
\hline Fungi & 4.9 & 5.1 & 5.1 & 5.2 & 0.19 & $<0.001$ & 0.002 & 0.036 \\
\hline
\end{tabular}

${ }^{1}$ Effect of treating by-product-rich concentrates with LAC (Trt), phosphorus supplementation (Phos), and their interaction (Trt $\times$ Phos).

${ }^{2} \mathrm{OTU}=$ operational taxonomic units. 
other Bacteroidales family within Bacteroidetes phylum 26 and $21 \%$ compared with the CON treatment $(P<$ 0.05). One Tenericutes family each within the order RF39 and the class Mollicutes increased and decreased with the LAC treatment by 15 and $47 \%$ compared with the CON treatment, respectively $(P<0.05)$. Moreover, the family $R 4-41 B$ (100\% increase; phylum Verrucomicrobia), an unclassified Endomicrobia family (191\% increase) within the Elusibacteria and the families Dethiosulfovibrionaceae (25\% increase) and Synergistaceae (25\% decrease; phylum Synergistetes), the family R4-45B (20\% decrease; Lentisphaerae) and unclassified families within the class Alphaproteobacteria (61\% decrease), and the Proteobacteria order GMD14H09 (155\% increase) and Burkholderiales (117\% increase) were modified by the LAC treatment of BPC $(P<$ $0.05)$.

Inorganic $\mathrm{P}$ supplementation decreased $(P<0.05)$ the relative abundance of 2 unclassified Clostridiales families (14 and 49\% decrease, respectively) and 1 Clostridiaceae family (36\% decrease), 1 Tenericutes family within the order RF39 (14\% decrease), 3 low abundant Proteobacteria (Enterobacteriaceae, $32 \%$ decrease; Burkholderiales, 51\% decrease; and PB19, 54\% decrease), as well as of F16 within the phylum TM7, 1 Clorophlexi (Anaerolinaceae), and 1 Plantomycetes family (Pirellulaceae). By contrast, 9 families increased with the higher ruminal $\mathrm{P}$ availability of which 3 belonged to the phylum Proteobacteria (RF32 family, 31\% increase; Desulfovibrionaceae, $121 \%$ decrease; and a Rickettsiales family, $41 \%$ increase), 2 to Tenericutes (20 and $25 \%$ increase, respectively), and one each to the phyla Firmicutes (Erysipelotrichaceae, 39\% increase), Actinobacteria (Coriobacteriaceae, $41 \%$ increase), WPS-2 (unclassified family, $70 \%$ increase), and Armatimonadetes (unclassified RB046 family, $40 \%$ increase).

Lactic acid $\times$ inorganic $\mathrm{P}$ interactions $(P<0.05)$ indicated that the effect of LAC on Ruminococcaceae and Clostridiales family 1 was more pronounced in fermentors without the additional inorganic $\mathrm{P}$, whereas for Enterobacteriaceae the LAC effect was more distinct in fermentors with the inorganic $\mathrm{P}$ supplementation. Moreover, alterations in the relative abundances of the most abundance OTU caused by the LAC treatment of $\mathrm{BPC}$ and inorganic $\mathrm{P}$ supplementation reflected the observations made at higher taxonomic level, which are presented in Supplemental Table S2 (https://doi.org/ 10.3168/jds.2018-14821).

\section{Discriminant Bacterial Populations and Predicted Functions}

Multi-group sPLS-DA, a supervised classification method within the DIABLO N-integration, was ap-

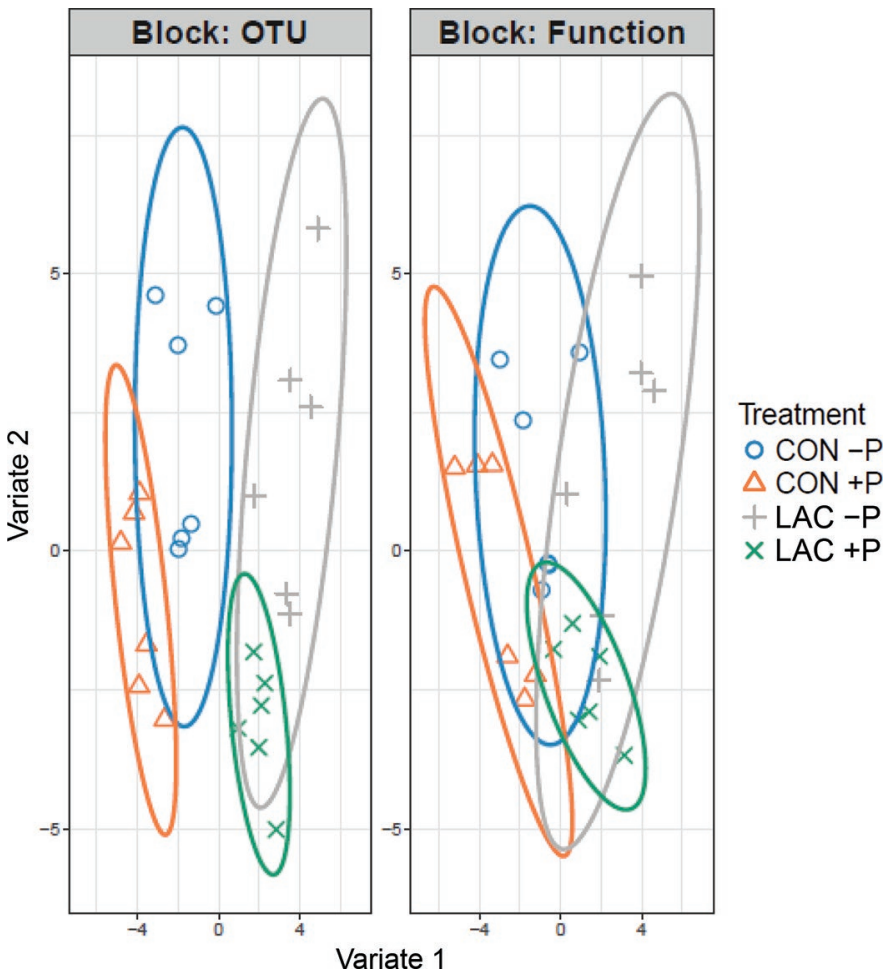

Figure 2. Scatter plots of horizontal sparse partial least squaresdiscriminant analysis for fermentor fluid samples with confidence ellipse plots. Block: OTU = scatter plot for operational taxonomic units (OTU); Block: Function $=$ scatter plot for predicted metagenome function. Treatment groups: $\mathrm{CON}=$ control-treated by-product-rich concentrate diet; $\mathrm{LAC}=5 \%$ lactic acid-treated $\mathrm{BPC}$ diet; $+\mathrm{P}=$ with inorganic $\mathrm{P}$ supplementation; $-\mathrm{P}=$ without inorganic $\mathrm{P}$ supplementation. Relative abundance of bacterial OTU and predicted Kyoto Encyclopedia of Genes and Genomes pathways $>0.01 \%$. Color version available online.

plied to identify the most precise OTU and predicted functional capabilities (KEGG pathways) that contributed to the discrimination for the component 1 and 2 between LAC versus CON treatment and between inorganic P supplementation versus without (Figure 2). Overall, the sPLS-DA sample plot indicated distinctive clustering between the LAC treatment and CON treatment of BPC, whereas the confidence intervals for the different inorganic $\mathrm{P}$ availabilities overlapped within the LAC and CON treatment. With regard to the predicted functional metagenome, there was no separate clustering among the treatment groups.

The differential abundances of the most discriminant OTU and KEGG pathways to components 1 and 2 are illustrated in Figures $3 \mathrm{~A}$ and 3B. Results from the univariate analysis for the single most abundant OTU as well as for the best discriminant OTU and predicted KEGG pathways can be found in Supplemental Tables S2, S3, S4, and S5 (https://doi.org/10.3168/jds.2018 -14821). Accordingly, 3 BS11-OTU (OTU21, OTU822, 
A

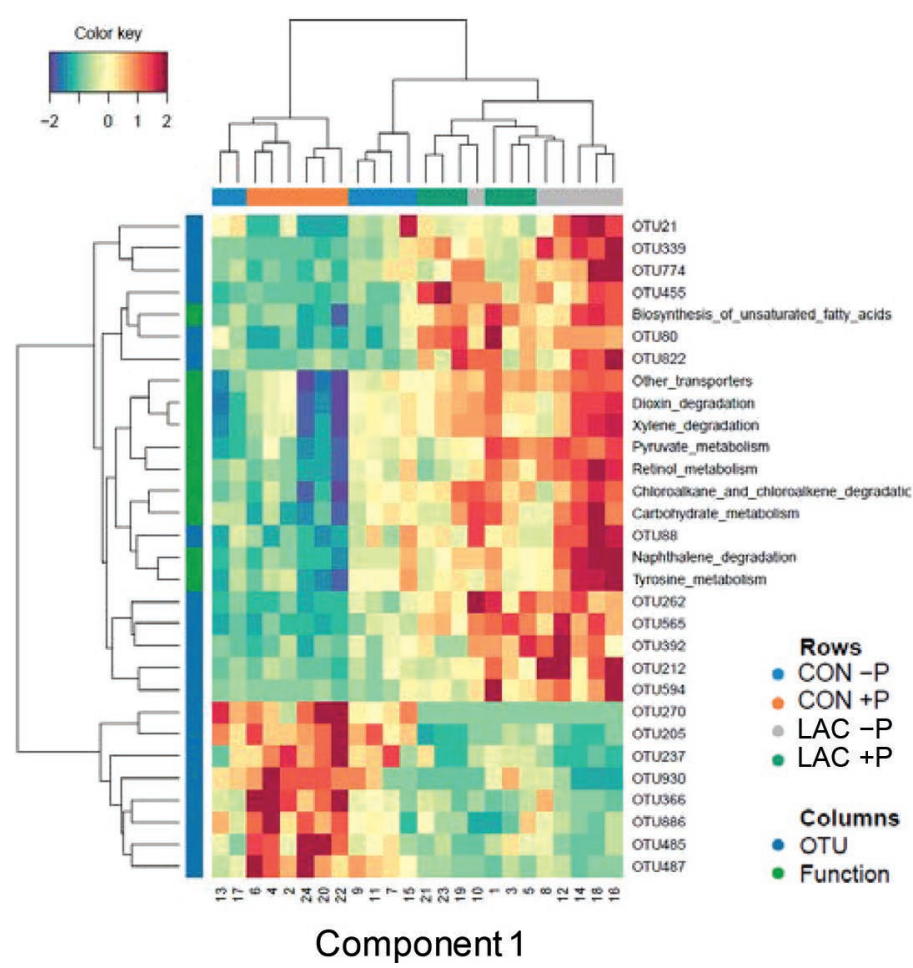

B

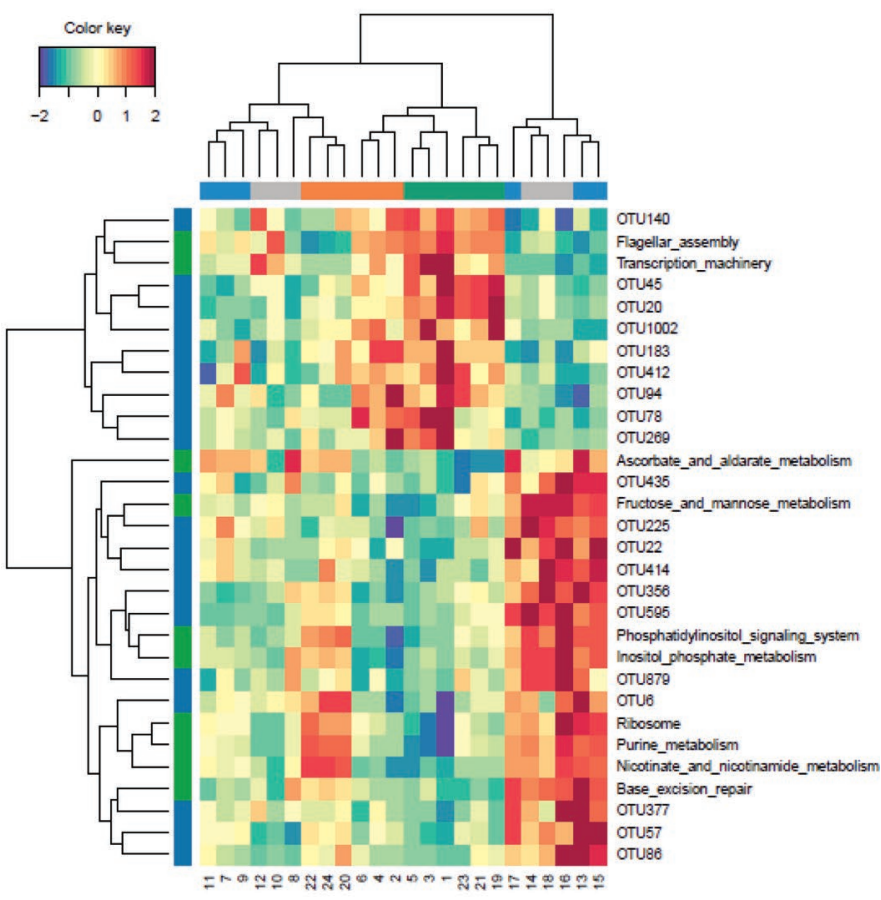

Component2

Figure 3. Clustered image maps (Euclidean distance, complete linkage) of the multi-omics signature for the identified best discriminant operational taxonomic units (OTU; $\mathrm{n}=20$ ) and predicted Kyoto Encyclopedia of Genes and Genomes $($ KEGG) pathways $(\mathrm{n}=10)$ showing different enrichment between the treatment groups for (A) component 1 and (B) component 2. Treatment groups: CON = control-treated byproduct-rich concentrate diet; $\mathrm{LAC}=5 \%$ lactic acid-treated by-product-rich concentrate diet; $+\mathrm{P}=$ with inorganic $\mathrm{P}$ supplementation; $-\mathrm{P}=$ without inorganic $\mathrm{P}$ supplementation. The $\mathrm{x}$-axis shows the fermentor fluid sample. The $\mathrm{y}$-axis presents the identified best discriminant $\mathrm{OTU}$ and predicted KEGG pathways per component. Relative abundance of bacterial OTU and predicted KEGG pathways $>0.01 \%$. Color version available online.

and OTU88), 2 RFN20-OTU (OTU339 and OTU565), 4 Ruminococcus-OTU (OTU774, OTU392, OTU212, and OTU594), 1 Ruminococcaceae-OTU (OTU80), 1 Anaerovibrio-OTU (OTU455), and 1 Christensenellaceae-OTU (OTU262), as well as all 10 discriminant KEGG pathways were enriched in ruminal fluid from fermentors that received the LAC-treated BPC for component 1 (Figure 3A; Supplemental Tables S3 and S4; https://doi.org/10.3168/jds.2018-14821). In contrast, 4 Bacteroidales-OTU (OTU270, OTU205, OTU485 and OTU487), 2 BS11-OTU (OTU366 and OTU866), 1 Clostridium-OTU (OTU930), 1 RF32-OTU (OTU237), and 1 ML615J-28-OTU (OTU86) were more abundant in the ruminal fluid from fermentors with the $\mathrm{CON}$ treatment for component 1 . For component 2 (Figure 3B; Supplemental Tables S3 and S4), 4 ClostridialesOTU (OTU94, OTU412, OTU45, and OTU20), 1 BS11-OTU (OTU78), and 3 Ruminococcaceae-OTU (OTU183, OTU140, and OTU269) were enriched in the fermentor fluid that received the treatments without additional inorganic P. Moreover, the KEGG pathways "phosphatidylinositol signaling system" and "inositol phosphate metabolism" were more enriched in fermentors with the supplemental inorganic $P$, whereas the KEGG pathway "transcription machinery" and "ribosome" were less abundant with more P.

\section{Associations Between Discriminant OTU and Imputed Bacterial Functions}

An integrative sPLS analysis was used to provide linear positive and negative relationships between discriminant OTU and imputed bacterial functional capabilities. Only the strongest correlations with $\mid \mathrm{r} />$ 0.80 were considered (Figures $4 \mathrm{~A}$ and $4 \mathrm{~B}$ ). For component 1 (Figure 4A), RFN20-OTU339 and Anaerovibrio-OTU455 were positively linked to the relative abundance of almost all discriminant KEGG pathways, whereas Bacteroidales-OTU270 and -OTU205 were negatively correlated with almost all discriminant KEGG pathways. Moreover, the relative abundance of 


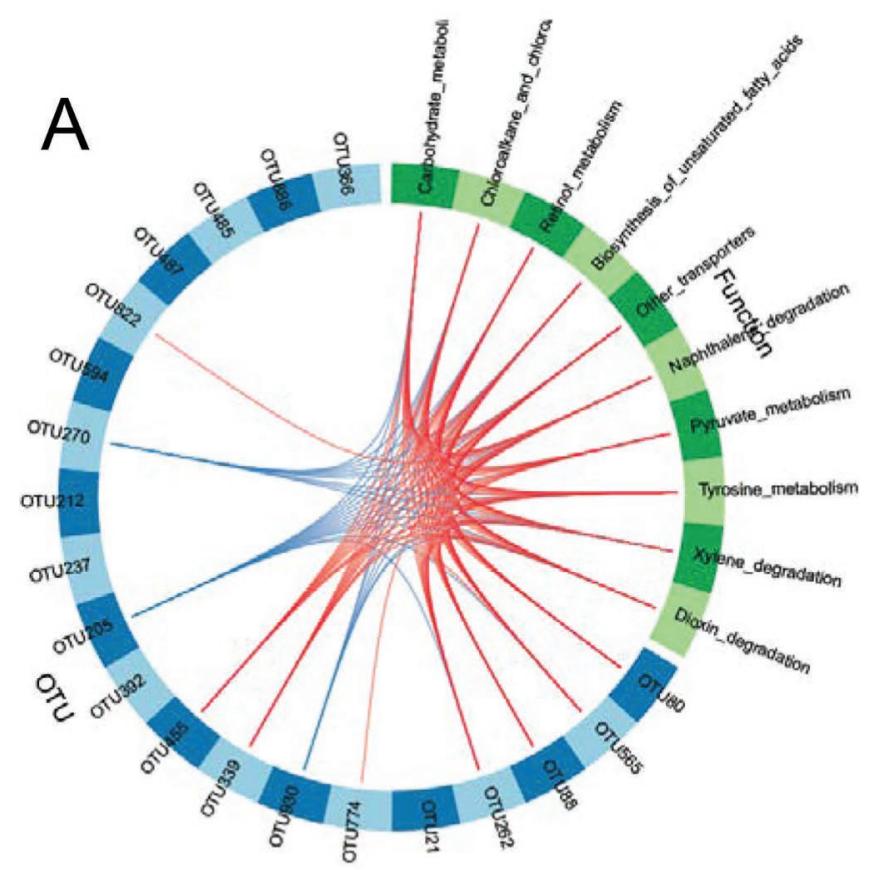

Component 1

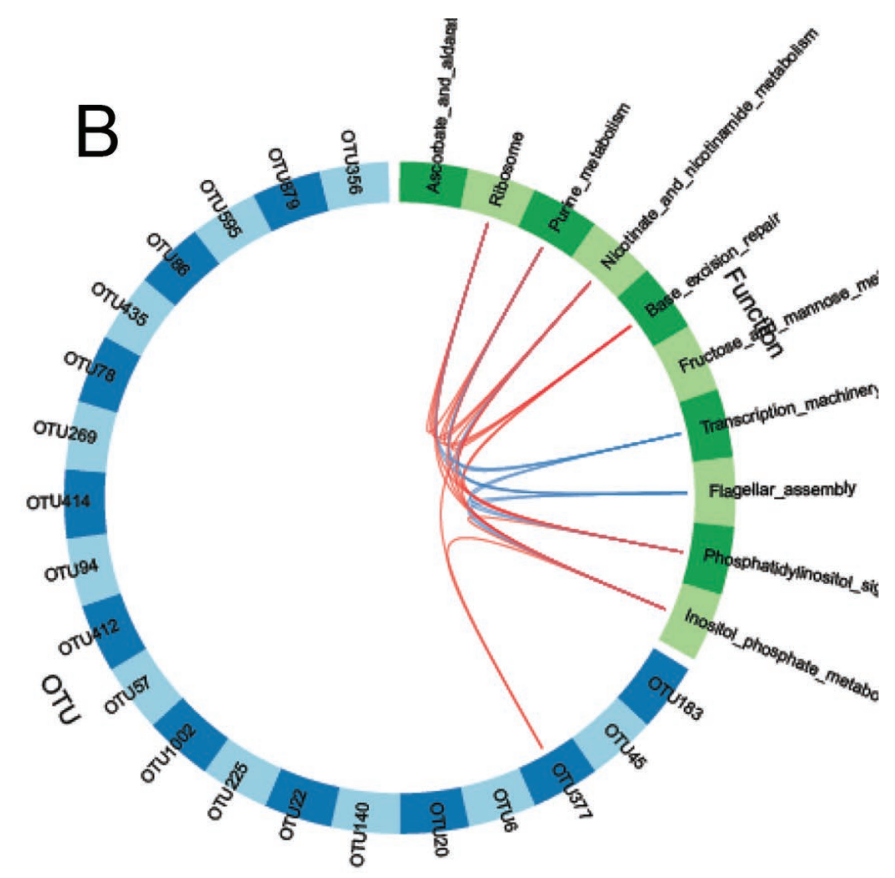

Component 2

Figure 4. Circos plots of horizontal sparse partial least squares-discriminant analysis displaying correlations $(\mid \mathrm{r} />0.8)$ between the identified best discriminant operational taxonomic units (OTU; $n=20)$ and predicted Kyoto Encyclopedia of Genes and Genomes (KEGG) pathways ( $\mathrm{n}$ $=10)$ for $(\mathrm{A})$ component 1 and $(\mathrm{B})$ component 2 . Positive and negative correlations $(\mid \mathrm{r} />0.8)$ are displayed by red and blue links, respectively. Relative abundance of bacterial OTU and predicted KEGG pathways $>0.01 \%$. Color version available online.

Christensenellaceae-OTU262, BS11-OTU88, RFN20OTU565, and Ruminococcaceae-OTU80 was positively associated with the KEGG pathways "carbohydrate metabolism," "pyruvate metabolism," and "biosynthesis of unsaturated fatty acids," whereas BacteroidalesOTU270 and Bacteroidales-OTU205, and ClostridiumOTU930 were negatively correlated with the KEGG pathways "carbohydrate metabolism" and "biosynthesis of unsaturated fatty acids." Christensenellaceae-OTU262 was further positively associated with tyrosine metabolism. The Ruminococcus-OTU774 and BS11-OTU822 were only positively correlated with the KEGG pathway "naphthalene degradation." Correlations among the KEGG pathways indicated positive dependencies of the KEGG pathways "carbohydrate metabolism" with "biosynthesis of unsaturated fatty acids," "other transporters," "naphthalene degradation," and "pyruvate metabolism." For component 2 (Figure 4B), fewer correlations $(|\mathrm{r}|>0.8)$ between discriminant OTU and KEGG pathways were found, mainly the positive association between the relative abundance of ClostridialesOTU377 and the KEGG pathways "inositol phosphate metabolism" and "nicotinate and nicotinamide metabolism." However, correlations between KEGG pathways showed that "inositol phosphate metabolism" positively correlated with "phosphatidylinositol signaling," "ribosome," "purine metabolism," and "base excision repair," whereas the KEGG pathway "transcription machinery" was negatively correlated with "ribosome," "purine metabolism," "inositol phosphate metabolism," and "phosphatidylinositol signaling."

\section{Pearson's Correlations of Discriminant OTU with Fermentation Characteristics}

Correlation analysis was used to estimate whether the most discriminate OTU were associated with changes in nutrient degradation and fermentation caused by the LAC treatment of BPC and inorganic P supplementation (Supplemental Figure S1; https://doi.org/10.3168/ jds.2018-14821). Accordingly, the strongest (negative) correlation with the daily $\mathrm{CO}_{2}$ production existed for RFN20-OTU57 ( $\mathrm{r}=-0.78 ; P<0.05)$. Strong positive correlations $(\mathrm{r}>0.75 ; P<0.05)$ existed between Clostridiales-OTU94 and Ruminococcaceae-OTU269 with the degradation of $\mathrm{OM}$ in the fermentor fluid. Moreover, Ruminococcaceae-OTU269 positively correlated $(\mathrm{r}=0.82 ; P<0.05)$ with the ruminal degradation of $\mathrm{ADF}_{\mathrm{OM}}$. The relative abundance of Desulfovibrionaceae-OTU225 and Clostridiales-OTU377, in turn, was 
negatively related to the ruminal disappearance of $\mathrm{P}(\mathrm{r}$ $<-0.75 ; P<0.05)$. The BS11-OTU6 strongly negatively correlated with the ruminal degradation of $\mathrm{OM}$ and the concentration of total SCFA and acetate in the fermentor fluid, whereas BS11-OTU14 negatively correlated with the total SCFA and propionate concentration $(\mathrm{r}<-0.75 ; P<0.05)$. Another BS11-OTU (OTU78) negatively correlated $(\mathrm{r}=-0.77 ; P<0.05)$ with the ruminal degradation of NFC. The relative abundance of RFN20-OTU57 was further negatively correlated with total SCFA, and RFN20-OTU356 and -OTU879 and Clostridiales-OTU595 with acetate $(\mathrm{r}<-0.75 ; P<0.05)$. Bacteroidales-OTU205 and -OTU270 were further negatively related to propionate, whereas Ruminococcaceae-OTU80, ChristensenellaceaeOTU262, Anaerovibrio-OTU455, and RFN20-OTU565 positively correlated with the propionate concentration in the fermentor fluid $(\mathrm{r}>0.75 ; P<0.05)$. In addition, Ruminococcaceae-OTU80 and Anaerovibrio-OTU455 positively correlated with butyrate and BacteroidalesOTU270 with isovalerate $(\mathrm{r}>0.75 ; P<0.05)$. The Christensenellaceae-OTU262 also negatively correlated $(\mathrm{r}=-0.76 ; P<0.05)$ with caproate. Moreover, the discriminant KEGG pathway "synthesis of unsaturated fatty acids" was positively correlated with propionate and butyrate, whereas the KEGG pathway "transcription machinery" was positively associated with total SCFA ( $>0.75 ; P<0.05$; Supplemental Figure S2; https://doi.org/10.3168/jds.2018-14821).

\section{DISCUSSION}

This study, based on $16 \mathrm{~S}$ rRNA gene sequencing of Rusitec fluid samples, demonstrated that treating BPC with LAC considerably altered the bacterial community composition and enhanced the bacterial richness and diversity, probably mirroring the changes in profiles and microbial degradability of nutrients in the LAC-treated compared with the CON-treated BPC. Besides, residual LAC may have served as substrate for LAC-utilizing bacteria (Flint et al., 2015). Especially taxa within Ruminococcaceae, an unclassified Clostridiales family, and Christensenellaceae benefited from the LAC treatment of BPC, having growth advantages as compared with taxa within the class Bacteroidales as shown by the integration network analysis. Although Megasphaera, as a well-known lactate utilizer (Flint et al., 2015), was not discriminant for the LAC treatment of BPC, lactate-utilizing Selenomonas became enriched in fermentors that received LAC-treated BPC.

Increasing the $\mathrm{P}$ availability with inorganic $\mathrm{P}$ in the fermentors further modulated the bacterial community composition, again predominantly taxa within the orders Bacteroidales and Clostridiales, and enhanced diversity. Univariate analysis showed that LAC-related increases and decreases in relative bacterial abundances were partly more distinguishable without the supplemental inorganic $\mathrm{P}$, supporting the notion that changes in the microbial $\mathrm{P}$ availability may have contributed to the shifts in bacterial abundances caused by the LAC treatment of $\mathrm{BPC}$. The inorganic $\mathrm{P}$ supplementation increased the available $\mathrm{P}$ for microbial growth in the fermentor with both CON- and LAC-treated BPC; hence, LAC-related effects may have been more related to changes in other nutrients. As indicated by the overlapping $95 \%$ confidence intervals in the sample plot, the changes in the imputed metabolic functions were not as drastic as observed for the bacterial community structure. Both LAC treatment of BPC and the supplemented inorganic $\mathrm{P}$ influenced the ruminal fermentation but in opposite directions. Increased SCFA, especially propionate, with the LAC-treated BPC may be favorable in the nutrition of high-yielding dairy cows, providing precursors for gluconeogenesis (Overton and Waldron, 2004). In contrast, the extra P may depress the ruminal energy supply by decreasing nutrient degradation and SCFA generation. This is an interesting finding because the inorganic $\mathrm{P}$ is commonly supplemented to diets with total $\mathrm{P}$ levels meeting cattle's $\mathrm{P}$ requirement to avoid depression of fiber degradation (Humer and Zebeli, 2015).

Because this study used an in vitro system and next-generation sequencing, an indirect and semiquantitative technology to estimate the various bacterial populations in the fermentor fluid, associations of the best discriminant bacterial populations with nutrient degradation, SCFA, and imputed functions are an approximation. More specifically, we analyzed the bacterial microbiome of the liquid-phase bacteria only, not the important solid-phase community. Due to bacterial species and functional differences, the results obtained for the liquid phase cannot be extrapolated to the solid phase. This needs to be considered when interpreting the present data.

Although total bacterial, archaeal, and protozoal gene copy numbers were equal among treatment groups, the increased bacterial species richness and evenness with the LAC-treated BPC may have indicated that more bacteria were able to use the $\mathrm{BPC}$ or, via cross-feeding, primary degradation metabolites as substrate. Acidic hydrolysis followed by leaching of nutrients into the soaking medium, however, may have lessened the microbial availability of certain nutrients, such as ash including P, CP, and released sugars from the hydrolysis of pectins and other hemicelluloses (Metzler-Zebeli et al., 2014; Harder et al., 2015). In line with that, soaking of BPC in LAC may have mainly hydrolyzed the soluble protein fraction. Consequently, less degradable 
protein remained as substrate for bacterial growth with the LAC-treated BPC, probably explaining the decreased CP degradation, lower proportion of branchedchain fatty acids, and changes in relative abundances of bacterial groups with proteolytic abilities, including taxa within the orders RF32, Bacteroidales, and Clostridiales. By increasing the UDP fraction in the LACtreated $\mathrm{BPC}$ diet, the ruminal degradability of $\mathrm{CP}$ is lowered, which may be attractive in feed formulation for high-yielding dairy cattle (Westreicher-Kristen et al., 2012).

In contrast to soaking of barley in $5 \%$ LAC where $\mathrm{aNDF}_{\mathrm{OM}}$ and $\mathrm{ADF}_{\mathrm{OM}}$ became enriched (Metzler-Zebeli et al., 2014; Harder et al., 2015), both fiber fractions were reduced in the LAC-treated BPC without affecting their degradability in the fermentors. As low $\mathrm{pH}$ already triggers their hydrolysis, the $\mathrm{pH}$ of 1.8 of the 5\% LAC solution may have been low enough to start the hydrolysis of the $\alpha$ - and $\beta$-glycosidic bonds in the hemicellulose fractions, such as arabinoxylan, arabinomannans, and $\beta$-glucans, and even of cellulose as $\mathrm{ADF}_{\mathrm{OM}}$ was less in the LAC-treated BPC (Krall and McFeeters, 1998; Kivelä et al., 2009). This will not only have affected the amount of fiber but also the fiber profile in the LAC-treated BPC, with consequences for the microbial composition, diversity, and metabolic activity. Our finding of a decreased acetate-to-propionate ratio and together with an increased daily production of $\mathrm{CH}_{4}$ and $\mathrm{CO}_{2}$ may support this reasoning. The elevated total SCFA concentration and the enrichment of the predicted KEGG pathways "carbohydrate metabolism," "pyruvate metabolism," and "biosynthesis of unsaturated fatty acids" further support an overall stimulation of microbial carbohydrate metabolism with the LAC-treated BPC diet. Although we observed an increase in the NFC fraction of the LAC-treated BPC and an enhanced NFC degradation in the respective fermentors, these data are speculative considering that the NFC is an indirect value and estimated by subtracting the contents of $\mathrm{CP}$, ether extract, and $\mathrm{aNDF}_{\mathrm{OM}}$ from the OM content.

Fungi and hemicellulolytic and cellulolytic members within the class Clostridiales, including the Ruminococcaceae family, may have profited from the changes in the fiber fractions of the LAC-treated BPC. Especially Clostridiales-OTU94 and -OTU269 seemed to be important for $\mathrm{aNDF}_{\mathrm{OM}}$ and $\mathrm{ADF}_{\mathrm{OM}}$ degradation and acetate production among the most discriminant OTU according to the positive correlations $(\mathrm{r} \geq 0.60)$. Interestingly, sPLS regression identified 5 OTU with identity to Ruminococcus bromii, a highly specialized amylolytic bacterium (Ze et al., 2015), as discriminant for the LAC-treated BPC diet. Due to the resemblance of 92 to $95 \%$ to cultivated strains and the low starch content of the diets, it may be more plausible that those OTU represented other Ruminococcus species, either benefiting from the LAC or modified hemicellulose fractions of the LAC-treated BPC. The same may be true for discriminative OTU, which were enriched with the LAC representing Selenomonas and Eubacterium species as both groups are capable of utilizing hemicelluloses and LAC (Cibis et al., 2016; Hall and Weimer, 2016). Likewise, the most abundant Ruminococcaceae-, Clostridiales- and Veillonellaceae-OTU, showing identity to Intestinimonas, Christensenella, and Anaerovibrio, may have had growth advantages only from the external LAC. Although the BS11 family was decreased with the LAC treatment of BPC, some OTU within this family may have benefited from the LAC modification, thereby contributing to $\mathrm{OM}$ and $\mathrm{P}$ degradation and SCFA production in the fermentor fluid as indicated by the positive correlations $(r \geq 0.57)$. This was supported by the sPLS regression, which identified OTU with identity to Selenomonas, Oxobacter, Eubacterium, Clostridium, Christensenellaceae, and BS11 as pivotal for the increased abundance of the KEGG pathways "carbohydrate metabolism," "pyruvate metabolism," and "biosynthesis of unsaturated fatty acids." However, the low taxonomic resolution rendered it difficult to infer specific metabolic abilities. In line with that, the $B S 11$ family has been poorly described in rumen microbiome surveys in cattle so far, which may be owed to the poor recognition by some $16 \mathrm{~S}$ rRNA databases (Quast et al., 2013; Solden et al., 2017). According to recent genome and proteome data, this taxonomic group is involved in SCFA production from hemicellulosic sugar fermentation (Solden et al., 2017). Despite often representing a large fraction of the rumen microbiota (Metzler-Zebeli et al., 2014), Prevotellaceae were not among the predominant taxa in the present study, which may be related to the fact that we only analyzed the bacterial community of the liquid phase and possibly also to the feeding of the donor cows and the time point of the sampling of the rumen inoculum. In general, certain shifts in the microbiota over time occur in the Rusitec system, which can lead to the depletion or enrichment of certain members compared with the original ruminal fluid. For this reason, the presently observed effects of the LAC-treated BPC and P supplementation should be verified in vivo.

Being essential for microbial growth, free phosphate concentrations in the rumen can limit fiber degradation if they become too low (Durand and Komisarczuk, 1988). In the present study, the total dietary $\mathrm{P}$ surpassed the current $\mathrm{P}$ recommendations of dairy cattle (3.2 to $3.8 \mathrm{~g}$ of $\mathrm{P} / \mathrm{kg}$ of $\mathrm{DM}$; NRC, 2001). However, because the $\mathrm{P}$ originated from plant material only in fermentors without the inorganic $\mathrm{P}$, treating phytate- 
rich $\mathrm{BPC}$ with LAC may have rendered the $\mathrm{P}$ in $\mathrm{BPC}$ more available to the microbes (Metzler-Zebeli et al., 2014). An enhanced $P$ availability may have therefore supported the growth and activity of hemicellulolytic and cellulolytic species (e.g., anaerobic fungi and Ruminococcaceae). However, leaching likely led to the lower $\mathrm{P}$ level in the LAC-treated BPC diet, thereby lowering the microbially available $\mathrm{P}$ in the LAC-treated $\mathrm{BPC}$ diet.

Bacteria within all dominant phyla benefited from the inorganic $\mathrm{P}$ supplementation, which was also reflected by the enrichment of the great majority of the most discriminant KEGG pathways, indicating an upregulation of metabolic processes. This disagreed with the depressed nutrient degradation, ammonia and SCFA concentrations, and gas production as well as higher redox potential in fermentors with the inorganic $\mathrm{P}$ supplementation. Several explanations for this scenario are possible. First, excessive $\mathrm{P}$ can be stored in the form of polyphosphates in the bacterial cell to be used as a source of energy (Wood and Clark, 1988). With the additional inorganic $\mathrm{P}$, it may be therefore conceivable that rumen microbes may have synthesized polyphosphates as an easily accessible source for ATP generation. Because these anabolic steps are metabolically costly, the transcription machinery for synthesis of proteases and carbohydrases may have been downregulated. This would be supported by the decreased relative abundance of the corresponding KEGG pathway "transcription machinery" and its negative relationship with most of the other key pathways identified for component 2. As indicated by the little correlations between the most discriminant OTU and KEGG pathways in the integration network, changes in the imputed metabolic functions were not associated with specific taxa but seemed to be related to a general shift in bacterial metabolism. Second, bacterial taxa with greater $\mathrm{P}$ requirements may have become enriched (Metzler-Zebeli et al., 2010), which may be behind the increase in Erysipelotrichaceae (RFN20), BS11 family, and proteolytic Desulfovibrionaceae. Notably, certain hemicellulolytic and cellulolytic bacteria, including Ruminococcaceae and unclassified Clostridiales-OTU, with potentially hemicellulolytic and cellulolytic capabilities and consequently higher $\mathrm{P}$ needs (Durand and Komisarczuk, 1988), were depressed by the higher $\mathrm{P}$ availability in the fermentors. It remains open whether this was a direct effect of the high environmental $\mathrm{P}$ concentration or due to microbe-microbe interactions, thereby limiting their proliferation.

In conclusion, soaking BPC in 5\% LAC may enhance microbial metabolic activity, which may be due to LAC-related changes in nutrient degradability. This was supported by considerable alterations in bacterial community composition, especially within the orders Bacteroidales and Clostridiales and enhanced relative abundance of imputed KEGG pathways related to carbohydrate metabolism. Because some effects of the LAC treatment were more pronounced without the additional inorganic $\mathrm{P}$, shifts in the bacterial community may be related to an altered microbial $\mathrm{P}$ availability with the LAC-treated BPC. Although adding inorganic $\mathrm{P}$ to the diets of high-producing cattle that otherwise meet the host and bacterial $\mathrm{P}$ needs is common practice, the present data of rumen nutrient degradation, fermentation, and fibrolytic bacteria, however, indicate that this supplementation above the requirements may harm rumen nutrient utilization. Because these results were produced from the liquid phase only using an in vitro system, the present findings need to be verified in vivo.

\section{ACKNOWLEDGMENTS}

The research was part of the project "Optimization of rations for dairy cows to reduce the use of foods potentially edible to humans (cereal grains, pulses) under Austrian organic agriculture conditions" funded by The Austrian Federal Ministry of Agriculture, Food and Environment (project number 100881).

\section{REFERENCES}

Benjamini, Y., and Y. Hochberg. 1995. Controlling the false discovery rate: A practical and powerful approach to multiple testing. J. R. Stat. Soc. B 57:289-300.

Caporaso, J. G., J. Kuczynski, J. Stombaugh, K. Bittinger, F. D. Bushman, E. K. Costello, N. Fierer, A. G. Peña, J. K. Goodrich, J. I. Gordon, G. A. Huttley, S. T. Kelley, D. Knights, J. E. Koenig, R. E. Ley, C. A. Lozupone, D. McDonald, B. D. Muegge, M. Pirrung, J. Reeder, J. R. Sevinsky, P. J. Turnbaugh, W. A. Walters, J. Widmann, T. Yatsunenko, J. Zaneveld, and R. Knight. 2010. QIIME allows analysis of high-throughput community sequencing data. Nat. Methods 7:335-336.

Cibis, K. G., A. Gneipel, and H. König. 2016. Isolation of acetic, propionic and butyric acid-forming bacteria from biogas plants. J. Biotechnol. 220:51-63.

Clark, W. D., J. Wohlt, R. Gilbreath, and P. Zajac. 1986. Phytate phosphorus intake and disappearance in the gastrointestinal tract of high producing dairy cows. J. Dairy Sci. 69:3151-3155.

Cole, J. R., Q. Wang, E. Cardenas, J. Fish, B. Chai, R. J. Farris, A. S. Kulam-Syed-Mohideen, D. M. McGarrell, T. Marsh, G. M. Garrity, and J. M. Tiedje. 2009. The Ribosomal Database Project: Improved alignments and new tools for rRNA analysis. Nucleic Acids Res. 37:D141-D145. https://doi.org/10.1093/nar/gkn879.

Deckardt, K., B. U. Metzler-Zebeli, and Q. Zebeli. 2016. Processing barley grain with lactic and tannic acid ameliorates rumen microbial fermentation and degradation of dietary fibre in vitro. J. Sci. Food Agric. 96:223-231. https://doi.org/10.1002/jsfa.7085.

Durand, M., and S. Komisarczuk. 1988. Influence of major minerals on rumen microbiota. J. Nutr. 118:249-260.

Edgar, R. C. 2010. Search and clustering orders of magnitude faster than BLAST. Bioinformatics 26:2460-2461.

Edgar, R. C., B. J. Haas, J. C. Clemente, C. Quince, and R. Knight. 2011. UCHIME improves sensitivity and speed of chimera detection. Bioinformatics 27:2194-2200. 
Ertl, P., W. Knaus, and W. Zollitsch. 2016a. An approach to including protein quality when assessing the net contribution of livestock to human food supply. Animal 10:1883-1889.

Ertl, P., Q. Zebeli, W. Zollitsch, and W. Knaus. 2016b. Feeding of wheat bran and sugar beet pulp as sole supplements in high-forage diets emphasizes the potential of dairy cattle for human food supply. J. Dairy Sci. 99:1228-1236.

Federal Ministry of Health. 2004. Verordnung der Bundesministerin für Gesundheit und Frauen über die Mindestanforderungen für die Haltung von Pferden und Pferdeartigen, Schweinen, Rindern, Schafen, Ziegen, Schalenwild, Lamas, Kaninchen, Hausgeflügel, Straußen und Nutzfischen (1. Tierhaltungsverordnung) StF: BGBl. II Nr. 485/2004.

Feng, X., K. Knowlton, A. Dietrich, and S. Duncan. 2013. Effect of abomasal ferrous lactate infusion on phosphorus absorption in lactating dairy cows. J. Dairy Sci. 96:4586-4591.

Flint, H. J., S. H. Duncan, K. P. Scott, and P. Louis. 2015. Links between diet, gut microbiota composition and gut metabolism. Proc. Nutr. Soc. 74:13-22. https://doi.org/10.1017/S0029665114001463.

Hall, M. B., and L. E. Chase. 2014. Responses of late-lactation cows to forage substitutes in low-forage diets supplemented with byproducts. J. Dairy Sci. 97:3042-3052.

Hall, M. B., and P. J. Weimer. 2016. Divergent utilization patterns of grass fructan, inulin, and other nonfiber carbohydrates by ruminal microbes. J. Dairy Sci. 99:245-257.

Harder, H., A. Khol-Parisini, B. U. Metzler-Zebeli, F. Klevenhusen, and Q. Zebeli. 2015. Treatment of grain with organic acids at 2 different dietary phosphorus levels modulates ruminal microbial community structure and fermentation patterns in vitro. J. Dairy Sci. 98:8107-8120.

Humer, E., and Q. Zebeli. 2015. Phytate in feed ingredients and potentials for improving the utilization of phosphorus in ruminant nutrition. Anim. Feed Sci. Technol. 209:1-15.

Khol-Parisini, A., E. Humer, Ö. Sizmaz, S. Abdel-Raheem, L. Gruber, J. Gasteiner, and Q. Zebeli. 2015. Ruminal disappearance of phosphorus and starch, reticuloruminal $\mathrm{pH}$ and total tract nutrient digestibility in dairy cows fed diets differing in grain processing. Anim. Feed Sci. Technol. 210:74-85.

Kivelä, R., L. Nyström, H. Salovaara, and T. Sontag-Strohm. 2009. Role of oxidative cleavage and acid hydrolysis of oat beta-glucan in modelled beverage conditions. J. Cereal Sci. 50:190-197.

Krall, S., and R. F. McFeeters. 1998. Pectin hydrolysis: Effect of temperature, degree of methylation, $\mathrm{pH}$, and calcium on hydrolysis rates. J. Agric. Food Chem. 46:1311-1315.

Langille, M. G. I., J. Zaneveld, J. G. Caporaso, D. McDonald, D. Knights, J. A. Reyes, J. C. Clemente, D. E. Burkepile, R. L. V. Thurber, R. Knight, R. G. Beiko, and C. Huttenhower. 2013. Predictive functional profiling of microbial communities using $16 \mathrm{~S}$ rRNA marker gene sequences. Nat. Biotechnol. 31:814-821.

McDougall, E. I. 1948. Studies on ruminant saliva. I. The composition and output of sheep's saliva. Biochem. J. 43:99-109.

Metzler-Zebeli, B. U., K. Deckardt, M. Schollenberger, M. Rodehutscord, and Q. Zebeli. 2014. Lactic acid and thermal treatments trigger the hydrolysis of myo-inositol hexakisphosphate and modify the abundance of lower myo-inositol phosphates in barley (Hordeum vulgare L.). PLoS One 9:e101166. https://doi.org/10.1371/ journal.pone.0101166.

Metzler-Zebeli, B. U., A. Khol-Parisini, L. Gruber, and Q. Zebeli. 2015. Microbial populations and fermentation profiles in rumen liquid and solids of Holstein cows respond differently to dietary barley processing. J. Appl. Microbiol. 119:1502-1514.

Metzler-Zebeli, B. U., W. Vahjen, T. Baumgärtel, M. Rodehutscord, and R. Mosenthin. 2010. Ileal microbiota of growing pigs fed dif- ferent dietary calcium phosphate levels and phytase content and subjected to ileal pectin infusion. J. Anim. Sci. 88:147-158.

Morse, D., H. Head, and C. Wilcox. 1992. Disappearance of phosphorus in phytate from concentrates in vitro and from rations fed to lactating dairy cows. J. Dairy Sci. 75:1979-1986.

Naumann, C., and R. Basler. 2012. Association of German Agricultural Analytic and Research Institutes. 2012. Die Chemische Untersuchung von Futtermitteln. 8. Erg. 2012. VDLUFA-Verlag, Darmstadt, Germany.

NRC. 2001. Nutrient Requirements of Dairy Cattle. 7th rev. ed. Natl. Acad. Press, Washington, DC.

Overton, T., and M. Waldron. 2004. Nutritional management of transition dairy cows: Strategies to optimize metabolic health. J. Dairy Sci. 87(E Suppl.):E105-E119.

Peterson, J., S. Garges, M. Giovanni, P. McInnes, L. Wang, J. A Schloss, V. Bonazzi, J. E. McEwen, K. A. Wetterstrand, C. Deal, C. C. Baker, V. Di Francesco, T. K. Howcroft, R. W. Karp, R. D. Lunsford, C. R. Wellington, T. Belachew, M. Wright, C. Giblin, H. David, M. Mills, R. Salomon, C. Mullins, B. Akolkar, L. Begg, C. Davis, L. Grandison, M. Humble, J. Khalsa, A. R. Little, H. Peavy, C. Pontzer, M. Portnoy, M. H. Sayre, P. Starke-Reed, S. Zakhari, J. Read, B. Watson, and M. Guyer. 2009. The NIH human microbiome project. Genome Res. 19:2317-2323.

Quast, C., E. Pruesse, P. Yilmaz, J. Gerken, T. Schweer, P. Yarza, J. Peplies, and F. O. Glöckner. 2013. The SILVA ribosomal RNA gene database project: Improved data processing and web-based tools. Nucleic Acids Res. 41:D590-D596.

Qumar, M., R. Khiaosa-ard, P. Pourazad, R. Aschenbach, U. Wetzels, F. Klevenhusen, and W. Kandler. 2016. Evidence of in vivo absorption of lactate and modulation of short chain fatty acid absorption from the reticulorumen of non- lactating cattle fed high concentrate diets. PLoS One 11:e0164192.

Rohart, F., B. Gautier, A. Singh, and K. A. Lê Cao. 2017. mixOmics: An $\mathrm{R}$ package for 'omics feature selection and multiple data integration. PLOS Comput. Biol. 13:e1005752. https://doi.org/10 .1371/journal.pcbi.1005752.

Solden, L. M., D. W. Hoyt, W. B. Collins, J. E. Plank, R. A. Daly, E. Hildebrand, T. J. Beavers, R. Wolfe, C. D. Nicora, S. O. Purvine, M. Carstensen, M. S. Lipton, D. E. Spalinger, J. L. Firkins, B. A. Wolfe, and K. C. Wrighton. 2017. New roles in hemicellulosic sugar fermentation for the uncultivated Bacteroidetes family BS11. ISME J. 11:691-703.

Van Soest, P. J., J. B. Robertson, and B. A. Lewis. 1991. Methods for dietary fiber, neutral detergent fiber, and nonstarch polysaccharides in relation to animal nutrition. J. Dairy Sci. 74:3583-3597.

Weatherburn, M. W. 1967. Phenol-hypochlorite reaction for determination of ammonia. Anal. Chem. 39:971-974.

Westreicher-Kristen, E., H. Steingass, and M. Rodehutscord. 2012. Variations in chemical composition and in vitro and in situ ruminal degradation characteristics of dried distillers' grains with solubles from European ethanol plants. Arch. Anim. Nutr. 66:458-472.

Wilkinson, J. M., and M. R. F. Lee. 2017. Review: Use of humanedible animal feeds by ruminant livestock. Animal 12:1735-1743. https://doi.org/10.1017/S175173111700218X.

Wood, H. G., and J. E. Clark. 1988. Biological aspects of inorganic polyphosphates. Annu. Rev. Biochem. 57:235-260.

Ze, X., Y. Ben David, J. A. Laverde-Gomez, B. Dassa, P. O. Sheridan, S. H. Duncan, P. Louis, B. Henrissat, N. Juge, N. M. Koropatkin, E. A. Bayer, and H. J. Flint. 2015. Unique organization of extracellular amylases into amylosomes in the resistant starch-utilizing human colonic firmicutes bacterium Ruminococcus bromii. MBio 6:e01058-15. 